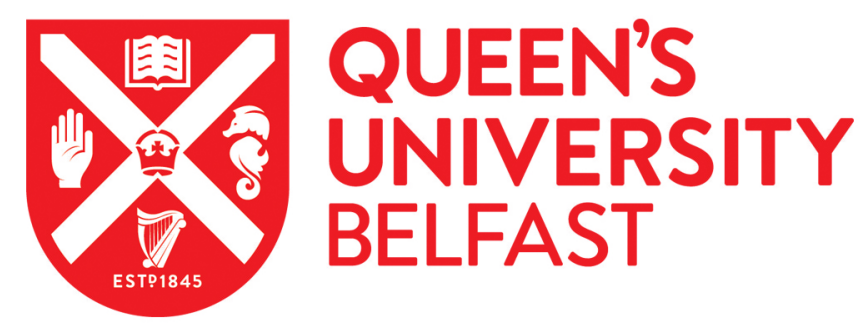

\title{
A Tractable Approach to Uplink Spectral Efficiency of Two-Tier Massive MIMO Cellular HetNets
}

Liu, W., Jin, S., Wen, C-K., Matthaiou, M., \& You, X. (2016). A Tractable Approach to Uplink Spectral Efficiency of Two-Tier Massive MIMO Cellular HetNets. IEEE Communications Letters, 20(2), 348-351.

https://doi.org/10.1109/LCOMM.2015.2503758

\section{Published in:}

IEEE Communications Letters

\section{Document Version:}

Peer reviewed version

Queen's University Belfast - Research Portal:

Link to publication record in Queen's University Belfast Research Portal

\begin{abstract}
Publisher rights
(c) 2015 IEEE. Personal use of this material is permitted. Permission from IEEE must be obtained for all other users, including reprinting/ republishing this material for advertising or promotional purposes, creating new collective works for resale or redistribution to servers or lists, or reuse of any copyrighted components of this work in other works
\end{abstract}

\section{General rights}

Copyright for the publications made accessible via the Queen's University Belfast Research Portal is retained by the author(s) and / or other copyright owners and it is a condition of accessing these publications that users recognise and abide by the legal requirements associated with these rights.

Take down policy

The Research Portal is Queen's institutional repository that provides access to Queen's research output. Every effort has been made to ensure that content in the Research Portal does not infringe any person's rights, or applicable UK laws. If you discover content in the Research Portal that you believe breaches copyright or violates any law, please contact openaccess@qub.ac.uk. 


\title{
A Tractable Approach to Uplink Spectral Efficiency of Two-Tier Massive MIMO Cellular HetNets
}

\author{
Wen Liu, Student Member, IEEE, Shi Jin, Member, IEEE, Chao-Kai Wen, Member, IEEE, \\ Michail Matthaiou, Senior Member, IEEE and Xiaohu You, Fellow, IEEE
}

\begin{abstract}
This letter investigates the uplink spectral efficiency (SE) of a two-tier cellular network, where massive multipleinput multiple-output macro base stations are overlaid with dense small cells. Macro user equipments (MUEs) and small cells with single user equipment uniformly scattered are modeled as two independent homogeneous Poisson point processes. By applying stochastic geometry, we analyze the SE of the multiuser uplink at a macro base station that employs a zero-forcing detector and we obtain a novel lower bound as well as its approximation. According to the simple and near-exact analytical expression, we observe that the ideal way to improve the $\mathrm{SE}$ is by increasing the MUE density and the base station antennas synchronously rather than increasing them individually. Furthermore, a large value of path loss exponent has a positive effect on the SE due to the reduced aggregated interference.
\end{abstract}

Index Terms-Massive MIMO, Poisson point process, small cells, spectral efficiency, stochastic geometry

\section{INTRODUCTION}

In the emerging $5 \mathrm{G}$ communication systems, massive multiple-input multiple-output (MIMO) and small cells are both regarded as critical components [1] and have been extensively studied in recent years. These two promising technologies can be combined when macro cells with massive MIMO base stations (BSs) are overlaid by dense small cells; such a deployment utilizes the excessive number of antennas at macro BS for managing interference meanwhile takes advantage of the smaller propagation distance in small cells to greatly improve the throughput performance of cell edge users and extend coverage [2].

In general, stochastic geometry provides a nature way of modeling the locations of users or BSs and such is particularly relevant to interference analysis [3]. Recent efforts $[4,5]$ have focused mainly on deriving expressions for the outage capacity and approximated distribution of the signalto-interference-and-noise ratio (SINR), typically by involving

Manuscript received August 23, 2015; accepted November 16, 2015. The work of W. Liu, S. Jin, M. Matthaiou and X. You was supported by the National Natural Science Foundation of China under Grants 61221002 , 61531011, 61450110445, and 61222102, the National High Technology Research and Development Program of China under Grant 2014AA01A704 and the International Science and Technology Cooperation Program of China under Grant 2014DFT10300. The work of C.-K. Wen was supported by the ITRI in Hsinchu, Taiwan, under Grant E352J33120.

W. Liu, S. Jin and X. You are with the National Mobile Communications Research Laboratory, Southeast University, Nanjing 210096, China (e-mail: newen@seu.edu.cn; jinshi@seu.edu.cn; xhyu@seu.edu.cn).

C.-K. Wen is with the Institute of Communications Engineering, National Sun Yat-sen University, Kaohsiung 80424, Taiwan (e-mail: ckwen@ieee.org).

M. Matthaiou is with the School of Electronics, Electrical Engineering and Computer Science, Queens University Belfast, Belfast BT7 1NN, U.K. (email:m.matthaiou@qub.ac.uk).

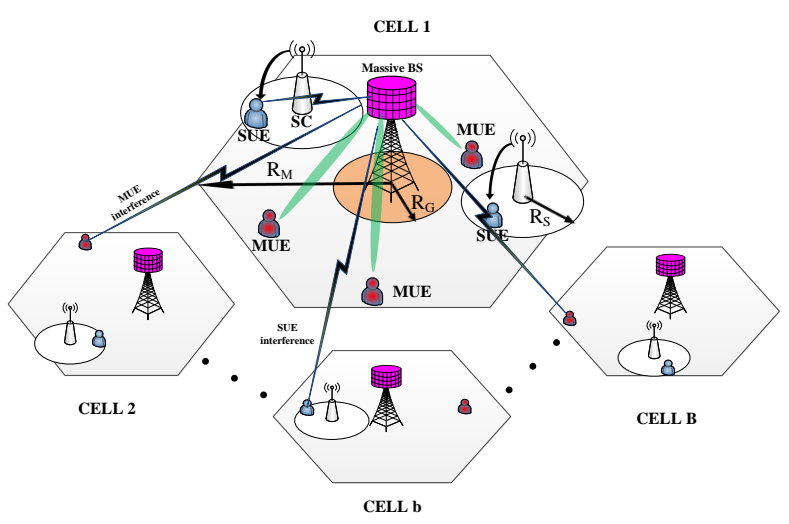

Fig. 1. Illustration of a two-tier network deployment with massive MIMO macro cells overlaid by small cells.

a Laplace transform in their final expressions. However, this mathematical technique blurs any engineering insights for network designers.

This paper considers a general two-tier cellular network model in which user equipments (UEs) and small cells are distributed as independent homogeneous Poisson point processes (PPPs). Given the analytical tractability of the PPP model, we derive a novel lower bound on the aggregate spectral efficiency (SE) and further obtain an approximated expression of this lower bound. With this concise expression, we can intuitionally determine how the model parameters influence the aggregate SE of the complex two-tier model.

Notations: The expectation and probability are denoted by $\mathbb{E}[\cdot]$ and $\mathbb{P}[\cdot]$, respectively. $\mathcal{C N}\left(0, \sigma^{2}\right)$ represents the complex Gaussian distribution with zero mean and variance of $\sigma^{2}$, while $\|\cdot\|$ denotes the Frobenius norm.

\section{SYSTEM MODEL}

We consider a cellular network consisting of $B$ (can be extended to infinity over the whole plane) macro tier massive MIMO BSs equipped with $M$ antennas, which is overlaid with small cells (SCs) each equipped with $N$ antennas, as explicitly shown in Fig. 1. Single-antenna UEs scattered over both tiers can be classified into two categories, namely, macro UEs (MUEs) and small cell UEs (SUEs), which are served by the BSs and SCs, respectively. This paper focuses on the uplink aggregate SE with fully frequency reuse in both tiers.

In the model of interest, BSs are located in the center of hexagonal cells, which can be approximated by a circular area for the sake of analytical tractability. The MUEs are randomly distributed in the range of the macro cell and can be modeled as a homogeneous PPP with density $\lambda_{\mathrm{m}}$. Meanwhile, the SCs 
are modeled as a homogeneous PPP with density $\lambda_{\mathrm{SC}}$. In addition, we assume that only one randomly distributed SUE is associated with each $\mathrm{SC}$ within its range in a resource block [2]. From the PPP property [6], we can easily deduce that the location of a SUE experiences an identical distribution with that of SCs, that is, the density of SUEs $\lambda_{\mathrm{s}}=\lambda_{\mathrm{SC}}$. The radii of BSs and SCs are defined as $R_{\mathrm{M}}$ and $R_{\mathrm{S}}$, respectively. In addition, the $\mathrm{BS}$ has a disk guard zone of radius $R_{\mathrm{G}}$, within which there are no UEs.

In the co-channel deployment, the $M \times 1$ received signal at the desired BS (i.e., the massive MIMO BS in cell 1) is

$$
\mathbf{y}=\sum_{b=1}^{B} \mathbf{G}_{b} \boldsymbol{\Lambda}_{b}^{1 / 2} \mathbf{x}_{b}+\sum_{b=1}^{B} \boldsymbol{\Omega}_{b} \mathbf{P}_{b}^{1 / 2} \mathbf{z}_{b}+\mathbf{n},
$$

where $\mathbf{G}_{b} \in \mathbb{C}^{M \times K_{b}}, \boldsymbol{\Omega}_{b} \in \mathbb{C}^{M \times S_{b}}$ denote the propagation matrices of the MUEs and SUEs in cell $b$ to the desired BS and $K_{b}, S_{b}$ are the total numbers of MUEs, SUEs in cell $b$. A typical propagation coefficient, for instance, $g_{b i s j}$, which defines the channel between the $i$ th BS antenna in the $b$ th cell and $j$ th UE in the sth cell, can be modeled as $g_{b i s j}=h_{b i s j} \beta_{b s j}^{1 / 2}$, where $h_{b i s j} \sim \mathcal{C N}(0,1)$ denotes the independent and identically distributed (i.i.d.) fast fading coefficients. The factor $\beta_{b s j}$, which represents large scale fading, is defined as $\beta_{b s j}=r_{b s j}^{-\alpha}$, where $\alpha$ is the path loss exponent. The large scale fading is assumed to be invariant between the two ends regardless of antenna difference [7]. In addition, $\boldsymbol{\Lambda}_{b}=\operatorname{diag}\left(\begin{array}{llll}p_{b 1}^{\mathrm{M}}, & p_{b 2}^{\mathrm{M}}, & \ldots, & p_{b K_{b}}^{\mathrm{M}}\end{array}\right)$ and $\mathbf{P}_{b}=\operatorname{diag}\left(p_{b 1}^{\mathrm{S}}, \quad p_{b 2}^{\mathrm{S}}, \quad \ldots, \quad p_{b S_{b}}^{\mathrm{S}}\right)$ are diagonal power matrices for MUEs and SUEs in cell $b ; \mathbf{x}_{b}$ and $\mathbf{z}_{b}$ represent the column transmit vectors of the MUEs and the SUEs to their associated BSs in cell $b$; and $\mathbf{n}$ models the complex addictive white Gaussian noise whose entries are i.i.d. with $\mathcal{C N}(0,1)$. For ease of notation, the transmit symbols are normalized as $\mathbb{E}\left[\mathbf{x}_{b} \mathbf{x}_{b}^{\mathrm{H}}\right]=\mathbf{I}_{K_{b}}, \mathbb{E}\left[\mathbf{z}_{b} \mathbf{z}_{b}^{\mathrm{H}}\right]=\mathbf{I}_{S_{b}}$.

Under the assumption of perfect CSI of its serving UEs, the linear zero-forcing (ZF) receiver is employed at the BSs. The received signal after the $\mathrm{ZF}$ detector is $\mathbf{r}=\mathbf{A}^{\mathrm{H}} \mathbf{y}$, where the $\mathrm{ZF}$ detection matrix is $\mathbf{A}=\mathbf{G}_{1}\left(\mathbf{G}_{1}^{\mathrm{H}} \mathbf{G}_{1}\right)^{-1}$. Then, the detected signal of the $k$ th MUE, which is equivalent to the $k$ th element of $\mathbf{r}$, can be further described as

$$
\begin{aligned}
r_{k}=\sqrt{p_{1 k}^{\mathrm{M}}} x_{1 k} & +\sum_{b=2}^{B} \sum_{c=1}^{K_{b}} \sqrt{p_{b c}^{\mathrm{M}}} \mathbf{a}_{k}^{\mathrm{H}} \mathbf{g}_{b c} x_{b c} \\
& +\sum_{b=1}^{B} \sum_{s=1}^{S_{b}} \sqrt{p_{b s}^{\mathrm{S}}} \mathbf{a}_{k}^{\mathrm{H}} \boldsymbol{\omega}_{b s} z_{b s}+\mathbf{a}_{k}^{\mathrm{H}} \mathbf{n},
\end{aligned}
$$

where $\mathbf{a}_{k}, \mathbf{g}_{b c}, \boldsymbol{\omega}_{b s}$ are the $k$ th, $c$ th, and $s$ th columns of $\mathbf{A}, \mathbf{G}_{b}, \boldsymbol{\Omega}_{b}$, respectively, and $p_{b c}$ denotes the uplink power of the $c$ th UE in the $b$ th cell. In this paper, we apply the Channel Inversion power control scheme, i.e., $p_{b c}=\beta_{b b c}^{-1}=r_{b b c}^{\alpha}$, to compensate for large scale fading. This power control scheme is widely used since it eliminates the near-far effect and guarantees the performance of cell edge UEs, only requiring long-term information [4]. Therefore, the SINR of the $k$ th MUE is written as

$$
\operatorname{SINR}_{k}=\frac{p_{1 k}^{\mathrm{M}}}{\sum_{b=2}^{B} \sum_{c=1}^{K_{b}} p_{b c}^{\mathrm{M}}\left|\mathbf{a}_{k}^{\mathrm{H}} \mathbf{g}_{b c}\right|^{2}+\sum_{b=1}^{B} \sum_{s=1}^{S_{b}} p_{b s}^{\mathrm{S}}\left|\mathbf{a}_{k}^{\mathrm{H}} \boldsymbol{\omega}_{b s}\right|^{2}+\left\|\mathbf{a}_{k}\right\|^{2}} .
$$

From (3), we observe that the MUE interference originates from inter-cells, whereas SUE interference originates from both intra- and inter-cells. The average SE of the $k$ th MUE can be obtained through

$$
R_{k}=\mathbb{E}\left[\log _{2}\left(1+\operatorname{SINR}_{k}\right)\right], \quad \text { (in bits/s/Hz) }
$$

where the expectation is taken over $\mathbf{a}_{k}, \mathbf{g}_{b c}$, and $\boldsymbol{\omega}_{b s}$.

\section{Spectral EfFiciency AnAlysis}

The average aggregate SE of MUEs in the desired cell is defined as

$$
R \triangleq \sum_{K_{1}=0}^{\infty} \mathbb{E}\left[\sum_{k=1}^{K_{1}} \log _{2}\left(1+\operatorname{SINR}_{k}\right) \mid \kappa=K_{1}\right] \cdot \mathbb{P}\left(\kappa=K_{1}\right),
$$

where $\kappa$, subject to a Poisson $\left(\mu_{\mathrm{m}}\right)$ distribution, denotes the random variable of the MUE number in the desired cell and $\mu_{\mathrm{m}}=\lambda_{\mathrm{m}} \mathcal{A}_{B S}$ denotes the mean number of MUEs, where $\lambda_{\mathrm{m}}$ is the MUE density and $\mathcal{A}_{B S}$ is the area of the macro cell. By substituting $\mathbb{P}\left(\kappa=K_{1}\right)=\frac{\mu_{\mathrm{m}}^{K_{1}} e^{-\mu_{\mathrm{m}}}}{K_{1} !}$ into (5), we derive

$$
R=\sum_{K_{1}=0}^{\infty} K_{1} \mathbb{E}\left[\log _{2}\left(1+\operatorname{SINR}_{k}\right) \mid \kappa=K_{1}\right] \cdot \frac{\mu_{\mathrm{m}}^{K_{1}} e^{-\mu_{\mathrm{m}}}}{K_{1} !},
$$

where we can extract $K_{1}$ from the expectation due to the fact that MUEs are generated from the same PPP.

To calculate the aggregate SE of MUEs, we have to determine the SE of a typical user, as expressed in (4). However, this task is extremely difficult to accomplish. As such, we seek a lower bound on the SE and its approximation in the following subsections.

\section{A. Lower Bound on the Spectral Efficiency}

First, we rewrite (3) into a concise form

$$
\mathrm{SINR}_{k}=\frac{p_{1 k}^{\mathrm{M}}}{(\underbrace{\sum_{i=1}^{N_{1}} p_{i}^{\mathrm{M}} \frac{\left|\mathbf{a}_{k}^{\mathrm{H}} \mathbf{g}_{i}\right|^{2}}{\left\|\mathbf{a}_{k}\right\|^{2}}+\sum_{j=1}^{N_{2}} p_{j}^{\mathrm{S}} \frac{\left|\mathbf{a}_{k}^{\mathrm{H}} \boldsymbol{\omega}_{j}\right|^{2}}{\left\|\mathbf{a}_{k}\right\|^{2}}}_{I}+1) \underbrace{\left\|\mathbf{a}_{k}\right\|^{2}}_{\chi_{0}}},
$$

where, for simplicity, we have reduced the subscripts of $p_{b c}^{\mathrm{M}}$, $p_{b s}^{\mathrm{S}}, \mathbf{g}_{b c}, \boldsymbol{\omega}_{b s}$, and let the total number of MUE and SUE interferers be $N_{1}=\sum_{b=2}^{B} K_{b}$ and $N_{2}=\sum_{b=1}^{B} S_{b}$, respectively. By applying Jensen's inequality to $\mathbb{E}\left[\log _{2}\left(1+\mathrm{SINR}_{k}\right) \mid \kappa=K_{1}\right]$ and using the fact that $I$ and $\chi_{0}$ are independent, we obtain

$\mathbb{E}\left[\log _{2}\left(1+\operatorname{SINR}_{k}\right) \mid \kappa=K_{1}\right] \geq \log _{2}\left(1+\frac{1}{\mathbb{E}[(I+1)] \mathbb{E}\left[\frac{\chi_{0}}{p_{1 k}^{M}}\right]}\right)$,

where the condition term is omitted for simplicity.

Assume that a UE is uniformly distributed in the circular area with radius $R$ but excluded from the guard zone with radius $R_{\mathrm{G}}$. Then, the probability density function (pdf) of the distance $r$ from UE to the center is given by

$$
f(r)= \begin{cases}\frac{2 r}{R^{2}-R_{\mathrm{G}}^{2}}, & r \in\left(R_{\mathrm{G}}, R\right], \\ 0, & \text { others. }\end{cases}
$$

Adopting this distance pdf, we have the following theorem.

Theorem 1: The lower bound on the aggregate $\mathrm{SE}$ in the desired cell is given below and $c_{\mathrm{GS}}=\frac{R_{\mathrm{G}}}{R_{\mathrm{S}}}, c_{\mathrm{GM}}=\frac{R_{\mathrm{G}}}{R_{\mathrm{M}}}$

$$
R_{\text {lower }}=\sum_{K_{1}=0}^{\infty} K_{1} \frac{\mu_{\mathrm{m}}^{K_{1}} e^{-\mu_{\mathrm{m}}}}{K_{1} !} \log _{2}\left(1+\frac{M-K_{1}}{I_{1}+I_{2}+1}\right),
$$


where $I_{1}=4 \pi \lambda_{\mathrm{m}} \frac{R_{\mathrm{M}}^{2}\left(1-c_{\mathrm{GM}}^{\alpha+2}\right)}{\left(\alpha^{2}-4\right)\left(1-c_{\mathrm{GM}}^{2}\right)}$ and $I_{2}=4 \pi \lambda_{\mathrm{s}} \frac{R_{\mathrm{S}}^{2} c_{\mathrm{GS}}^{2-\alpha}}{\alpha^{2}-4}$.

Proof: See Appendix A.

Theorem 1 shows how the aggregate SE scales with the system parameters, namely, the MUE density $\lambda_{\mathrm{m}}\left(\mu_{\mathrm{m}}\right.$ can also represent MUE density when area is a constant), the SUE density $\lambda_{\mathrm{s}}$, BS antenna number $M$, and the path loss exponent $\alpha$. These parameters will be discussed in detail in the next subsection by evaluating a more simplified expression.

\section{B. Approximated Lower Bound Analysis}

The lower bound expression (10) consists of the sum of infinite series, which is still difficult to evaluate. Alternatively, we notice that the variable of the summation $K_{1}$ only appears in the logarithmic function in the form of $M-K_{1}$. In the model of interest, we have $M \gg K_{1}$. Therefore, an approximation of the lower bound can be obtained by disregarding $K_{1}$, which results in the following corollary.

Corollary 1: The lower bound on the aggregate SE can be approximated by

$$
R_{\text {lower }} \approx \hat{R}_{\text {lower }}=\mu_{\mathrm{m}} \log _{2}\left(1+\frac{M}{\frac{4 \mu_{\mathrm{m}}}{\alpha^{2}-4}+4 \pi \lambda_{\mathrm{s}} \frac{R_{\mathrm{S}}^{2} c_{\mathrm{GS}}^{2-\alpha}}{\alpha^{2}-4}+1}\right) .
$$

Proof: As $M \gg K_{1}, c_{\mathrm{GM}} \ll 1$, we omit $K_{1}, c_{\mathrm{GM}}$ in (10) and obtain the final result with some simple algebra.

The approximation by disregarding $K_{1}$ renders a closedform expression without infinite series. This expression can be extremely close to $R_{\text {lower }}$ when $M$ becomes large. With the simple and clear expression of (11), we present some insights into properties of this two-tier network.

Corollary 2: As $\mu_{\mathrm{m}} \rightarrow \infty$, which states that the mean number of MUEs in each macro cell approaches infinity, the approximation of the lower bound is given by

$$
\left(\hat{R}_{\text {lower }}\right)_{\mu_{\mathrm{m}} \rightarrow \infty}=\frac{M\left(\alpha^{2}-4\right)}{4} \log _{2} e .
$$

Proof: Applying the fact that $\lim _{x \rightarrow \infty}\left(1+\frac{1}{x}\right)^{x}=e$ to (11), we obtain the desired result.

Remark 1: Several parameters in (11) provide opportunities to enhance the aggregate SE, such as increasing the MUE density. However, Corollary 2 suggests that the aggregate SE converges to a certain value in terms of $M$ and $\alpha$ when we densify MUE without constraint, which, however, diminishes the average SE of each MUE. Since dense deployment causes heavy interference, a tradeoff exists between the average SE and the aggregate SE. Therefore, after ensuring a certain average SE, the MUE density can be increased as far as possible to obtain the highest aggregate SE.

Remark 2: From (11), the aggregate SE can also be improved by increasing $M$. To this end, we take the derivative of (11) w.r.t. $M$ and obtain

$$
\tau_{M}=\frac{\mathrm{d} \hat{R}_{\text {lower }}}{\mathrm{d} M}=\frac{\mu_{\mathrm{m}} \log _{2} e}{\frac{4 \mu_{\mathrm{m}}}{\alpha^{2}-4}+4 \pi \lambda_{\mathrm{s}} \frac{R_{\mathrm{S}}^{2} c_{\mathrm{CS}}^{2-\alpha}}{\alpha^{2}-4}+1+M} .
$$

We find $\tau_{M} \rightarrow 0$ as $M \rightarrow \infty$. This means that if $\mu_{\mathrm{m}}$ and the other parameters are fixed, the slope of the aggregate SE decreases to zero. Therefore, by merely increasing the number of BS antennas we cannot avail of a sustainable growth of the aggregate SE because $M$ appears only inside the logarithm in
(11). By reforming (13), we find that the slope is determined by the ratio $\frac{M}{\mu_{\mathrm{m}}}$. Therefore, if $\mu_{\mathrm{m}}$ increases together with $M$ to infinity while keeping a fixed ratio $\frac{M}{\mu_{\mathrm{m}}}=c$, the slope does not converge to 0 but to

$$
\tau_{M} \rightarrow \frac{\log _{2} e}{\frac{4}{\alpha^{2}-4}+c} .
$$

This result supports the promising benefit of massive MIMO topologies in two-tier cellular networks.

\section{Numerical Results}

We consider a conventional seven-cell cellular network model. The parameters used in the simulations are summarized in Table I. All simulation points have been averaged over 100,000 different channel realizations and UE, SC locations. TABLE I

\section{Simulation PARAMETERS}

\begin{tabular}{|c||c|c|c|c|}
\hline Parameters & $R_{\mathrm{M}}$ & $R_{\mathrm{S}}$ & $R_{\mathrm{G}}$ & $\lambda_{\mathrm{s}}$ \\
\hline Values & $1000 \mathrm{~m}$ & $40 \mathrm{~m}$ & $40 \mathrm{~m}$ & $5 \times 10^{-5} \mathrm{~m}^{-2}$ \\
\hline
\end{tabular}

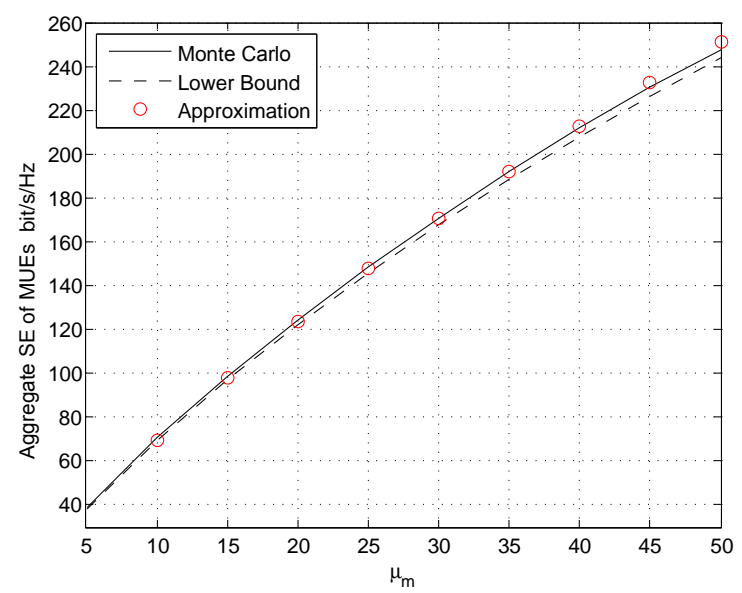

Fig. 2. The aggregate SE versus mean number of MUEs per cell with $\alpha=4$ and $M=500$ satisfying $M \gg K_{1}\left(\mu_{\mathrm{m}}\right)$.

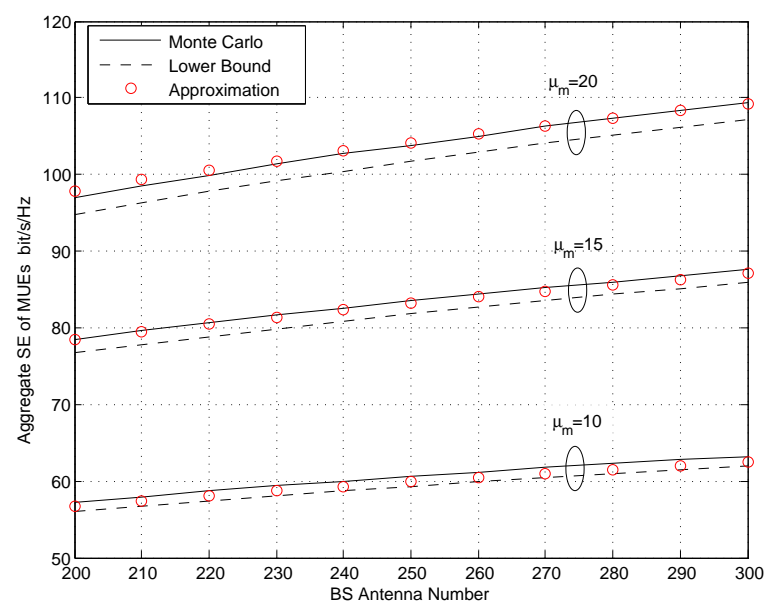

Fig. 3. The aggregate SE versus BS antenna number for different $\mu_{\mathrm{m}}$ with $\alpha=4$.

Fig. 2 illustrates the aggregate SE versus the mean number of MUEs per cell. The lower bound and its approximation are extremely tight with the Monte Carlo simulations, and the approximation is closer than the lower bound. The reason is that disregarding $K_{1}$ in (11) causes the approximation to be slightly larger than the lower bound, resulting in a more precise approximation of the lower bound. In addition, as shown in 


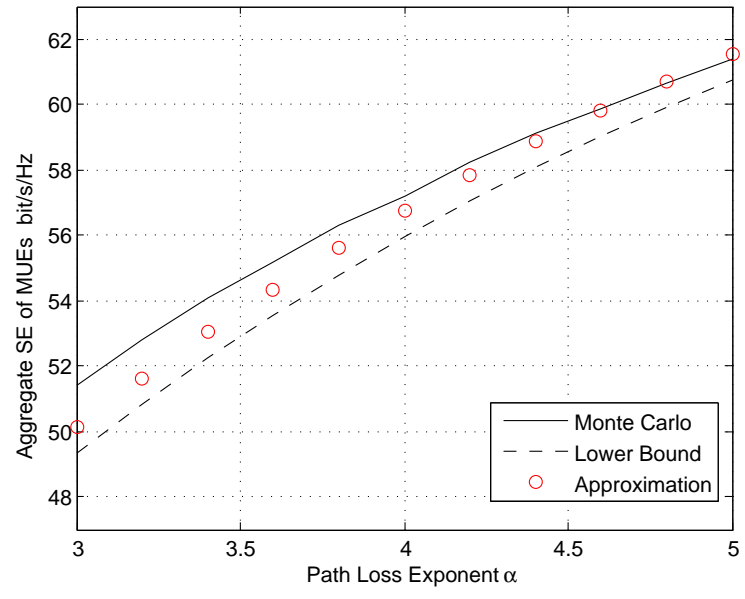

Fig. 4. The aggregate SE versus pass loss exponent $\alpha$ with $M=200$ and $\mu_{\mathrm{m}}=10$.

Fig. 2, accirding to the analysis of Corollary 2, the curves tend to flatten and will saturate when $\mu_{\mathrm{m}}$ becomes large enough.

Fig. 3 shows the aggregate SE performances with different $\mu_{\mathrm{m}}$ when the BS antenna number becomes large. We observe that the aggregate SE can be enhanced by increasing the BS antennas for all $\mu_{\mathrm{m}}$. The aggregate SE grows faster for the setting with the larger $\mu_{\mathrm{m}}$, as predicted by (14).

Fig. 4 depicts the aggregate SE as a function of the path loss exponent $\alpha$, which typically ranges from 3 to 5 . Interestingly, a larger $\alpha$ leads to a higher aggregate SE and a tighter bound. From (3), it can be observed that the useful signal power decreases less slowly than the aggregation of interference with increasing $\alpha$. Therefore, the aggregate SE for the scenario with higher path loss exponent outperforms that with a lower one.

\section{Conclusion}

This study investigated the aggregate uplink SE of MUEs in a two-tier network, and a novel lower bound, as well as a closed-form approximation, using tools of stochastic geometry. We also obtained new quantitative insights on how the system parameters influence the aggregate SE of this complex model. The proposed approach to SE can also be applied to the SE of SUEs, which will enable us to obtain the total SE of two-tier network in the future.

\section{APPENDIX A}

\section{PROOF OF THEOREM 1}

From (8), the problem of deriving the lower bound is now reduced to evaluating $\mathbb{E}[(I+1)]$ and $\mathbb{E}\left[\chi_{0} / p_{1 k}^{\mathrm{M}}\right]$. We first deal with the expectation of the aggregation interference. Thanks to Slivnyak's theorem [3], we can model MUE interferers as a homogeneous PPP $\Phi_{m}$ and SUE interferers as another independent homogeneous PPP $\Phi_{\mathrm{s}}$ without changing the density. From (7) and (8), we notice that the power, i.e., $p_{i}$, and the interfering channel vector are statistically independent. Therefore, the expectation of the aggregation interference is given by

$$
\mathbb{E}[I]=\mathbb{E}_{\Phi_{\mathrm{m}}}\left[\sum_{i} \mathbb{E}\left[p_{i}^{\mathrm{M}}\right] \mathbb{E}\left[\left|\hat{g}_{i}\right|^{2}\right]\right]+\mathbb{E}_{\Phi_{\mathrm{s}}}\left[\sum_{j} \mathbb{E}\left[p_{j}^{\mathrm{s}}\right] \mathbb{E}\left[\left|\hat{\omega}_{j}\right|^{2}\right]\right],
$$

where $\hat{g}_{i}=\frac{\mathbf{a}_{k}^{\mathrm{H}} \mathbf{g}_{i}}{\left\|\mathbf{a}_{k}\right\|}$ and $\hat{\omega}_{j}=\frac{\mathbf{a}_{k}^{\mathrm{H}} \boldsymbol{\omega}_{j}}{\left\|\mathbf{a}_{k}\right\|}$. Note that the entries of $\mathbf{g}_{i}$ and $\boldsymbol{\omega}_{j}$ are i.i.d. random variables with $\mathcal{C N}(0, \beta)$, where $\beta$ represents the large scale fading coefficient. In addition, $\hat{\mathbf{a}}_{k}=\frac{\mathbf{a}_{k}^{\mathrm{H}}}{\left\|\mathbf{a}_{k}\right\|}$ can be regarded as a location vector with $\left\|\hat{\mathbf{a}}_{k}\right\|^{2}=1$. Thus, with the independent nature between $\hat{\mathbf{a}}_{k}$ and $\left(\mathbf{g}_{i}, \boldsymbol{\omega}_{j}\right)$, together with the property that the sum of independent Gaussian variables is also Gaussian, we have $\hat{g}_{i} \sim \mathcal{C N}\left(0, \hat{\beta}_{i}\right)$ and $\hat{\omega}_{j} \sim \mathcal{C N}\left(0, \tilde{\beta}_{j}\right)$. In particular, we have

$$
\mathbb{E}\left[\left|\hat{g}_{i}\right|^{2}\right]=\hat{\beta}_{i}=\hat{r}_{i}^{-\alpha} \text { and } \mathbb{E}\left[\left|\hat{\omega}_{j}\right|^{2}\right]=\tilde{\beta}_{j}=\tilde{r}_{j}^{-\alpha} .
$$

Substituting the above and (9) into (15), we obtain

$$
\begin{aligned}
\mathbb{E}[I]=\mathbb{E}_{\Phi_{\mathrm{m}}}\left[\sum_{i}\left(\int_{R_{\mathrm{G}}}^{R_{\mathrm{M}}} l^{\alpha} \frac{2 l}{R_{\mathrm{M}}^{2}-R_{\mathrm{G}}^{2}} \mathrm{~d} l\right) \hat{r}_{i}^{-\alpha}\right] \\
+\mathbb{E}_{\Phi_{\mathrm{s}}}\left[\sum_{j}\left(\int_{0}^{R_{\mathrm{S}}} l^{\alpha} \frac{2 l}{R_{\mathrm{S}}^{2}} \mathrm{~d} l\right) \tilde{r}_{j}^{-\alpha}\right],
\end{aligned}
$$

where we have applied the channel inversion power control scheme. Then, we deal with the expectations over the sets $\Phi_{m}$ and $\Phi_{s}$. To this end, we apply the property of PPP [3, Lemma A.3.] and consider the interferers location range to determine the lower and upper limits of the integrals. Hence, we get

$$
\begin{aligned}
\mathbb{E}[I]= & \int_{R_{\mathrm{M}}}^{\infty} 2 \pi \lambda_{\mathrm{m}}\left(\int_{R_{\mathrm{G}}}^{R_{\mathrm{M}}} l^{\alpha} \frac{2 l}{R_{\mathrm{M}}^{2}-R_{\mathrm{G}}^{2}} \mathrm{~d} l\right) r^{-\alpha} \cdot r \mathrm{~d} r \\
& +\int_{R_{\mathrm{G}}}^{\infty} 2 \pi \lambda_{\mathrm{s}}\left(\int_{0}^{R_{\mathrm{S}}} l^{\alpha} \frac{2 l}{R_{\mathrm{S}}^{2}} \mathrm{~d} l\right) r^{-\alpha} \cdot r \mathrm{~d} r \\
= & 4 \pi \lambda_{\mathrm{m}} \frac{R_{\mathrm{M}}^{2}\left(1-c_{\mathrm{GM}}^{\alpha+2}\right)}{\left(\alpha^{2}-4\right)\left(1-c_{\mathrm{GM}}^{2}\right)}+4 \pi \lambda_{\mathrm{s}} \frac{R_{\mathrm{S}}^{2} c_{\mathrm{GS}}^{2-\alpha}}{\alpha^{2}-4},
\end{aligned}
$$

where the path loss exponent $\alpha>2$.

Next, we deal with $\mathbb{E}\left[\chi_{0} / p_{1 k}^{\mathrm{M}}\right]$. Recall that $\chi_{0}=\left\|\mathbf{a}_{k}\right\|^{2}=$ $\left[\mathbf{A}^{\mathrm{H}} \mathbf{A}\right]_{k k}=\left[\left(\mathbf{G}_{1}^{\mathrm{H}} \mathbf{G}_{1}\right)^{-1}\right]_{k k}$. Using the identity [8], we obtain the final result

$$
\mathbb{E}\left[\frac{\chi_{0}}{p_{1 k}^{\mathrm{M}}}\right]=\frac{1}{p_{1 k}^{\mathrm{M}} \beta_{11 k}\left(M-K_{1}\right)}=\frac{1}{M-K_{1}},
$$

where the second equality follows from $p_{1 k}^{\mathrm{M}}=r_{11 k}^{\alpha}=\frac{1}{\beta_{11 k}}$.

Finally, substituting (18), (19) into (8), we immediately arrive at the lower bound on the aggregate SE.

\section{REFERENCES}

[1] J. G. Andrews, S. Buzzi, W. Choi, S. Hanly, A. Lozano, A. C. Soong, and J. C. Zhang, "What will 5G be?" IEEE J. Sel. Areas Commun., vol. 32, no. 6, pp. 1065-1082, Jun. 2014.

[2] J. Hoydis, K. Hosseini, S. Ten Brink, and M. Debbah, "Making smart use of excess antennas: Massive MIMO, small cells, and TDD," Bell Labs Technical Journal, vol. 18, no. 2, pp. 5-21, Oct. 2013.

[3] M. Haenggi and R. K. Ganti, Interference in Large Wireless Networks. Now Publishers Inc, 2009.

[4] T. D. Novlan, H. S. Dhillon, and J. G. Andrews, "Analytical modeling of uplink cellular networks," IEEE Trans. Wireless Commun., vol. 12, no. 6 , pp. 2669-2679, Jun. 2013.

[5] R. W. Heath, Jr., M. Kountouris, and T. Bai, "Modeling heterogeneous network interference using Poisson point processes," IEEE Trans. Signal Process., vol. 61, no. 16, pp. 4114-4126, Aug. 2013.

[6] S. N. Chiu, D. Stoyan, W. S. Kendall, and J. Mecke, Stochastic Geometry and its Applications. John Wiley \& Sons, 2013.

[7] T. L. Marzetta, "Noncooperative cellular wireless with unlimited numbers of base station antennas," IEEE Trans. Wireless Commun., vol. 9, no. 11, pp. 3590-3600, Nov. 2010.

[8] Q. Zhang, S. Jin, K.-K. Wong, H. Zhu, and M. Matthaiou, "Power scaling of uplink massive mimo systems with arbitrary-rank channel means," IEEE J. Sel. Topics Signal Process., vol. 8, no. 5, pp. 966-981, 2014. 